\title{
The relationship of montreal cognitive assessment scores to framingham coronary and stroke risk scores
}

\author{
Myron Frederick Weiner, ${ }^{1,2 *}$, Linda Susan Hynan $^{1,3}$, Heidi Rossetti ${ }^{3}$, Matthew Wesley Warren ${ }^{1}$, \\ Colin Munro Cullum ${ }^{1,2}$
}

${ }^{1}$ Department of Psychiatry, University of Texas Southwestern Medical Center, Dallas, USA;

${ }^{2}$ Department of Neurology, University of Texas Southwestern Medical Center, Dallas, USA;

${ }^{3}$ Department of Clinical Sciences, University of Texas Southwestern Medical Center, Dallas, USA.

E-mail: *Myron.weiner@utsouthwestern.edu;

Received 3 June 2011; revised 25 June 2011; accepted 3 July 2011.

\begin{abstract}
We examined the relationship between a brief cognitive screening measure and Framingham Coronary and Stroke Risk scores. We administered the Montreal Cognitive Assessment (MoCA) to participants in the Dallas Heart Study, a community-based multiethnic study investigating the development of atherosclerosis. The composition of the group was $\mathbf{5 0 \%}$ African American, 36\% Caucasian and 14\% Hispanic. There were 765 subjects (mean age 51 years) who had both Coronary and Stroke Risk scores and an additional 144 subjects with only Coronary Risk scores available. There was a small significant inverse relationship between MoCA and Framingham Coronary and Stroke Risk scores. MoCA scores were influenced by education, but were not influenced by age or by the presence of one or more apoE4 alleles.
\end{abstract}

Keywords: Dementia; Montreal Cognitive Assessment; Cognition; Cardiovascular Risk

\section{INTRODUCTION}

Because of the strong evidence for a relationship between cardiovascular risk factors and cognitive compromise [1], the authors were asked to provide a brief measure of psychological function for the Dallas Heart Study (DHS), a population-based study of the development of atherosclerosis. Earlier studies of the relationship between environmental and biological factors and cognition in large population-based studies of elders have produced some positive findings [2,3]. Specifically, hypertension in late life has been associated with cognitive decline $[4,5]$ and thought possibly due to damaged brain vasculature. Additionally, increased total cholesterol has also been associated $[6,7]$ and thought to be due to brain lipid dysregulation, although it is not always shown [8]. Most studies of Type 2 diabetes have found a positive association between impaired glucose metabolism and dementia $[9,10]$. Taken together, metabolic syndrome with and without markers of inflammation have been associated with cognitive decline [11].

To facilitate participation, DHS subjects were seen in a single day that included a variety of biological measures. Because of the heavy schedule, DHS investigators required that our cognitive instrument be brief (15 minutes or less). We reviewed brief instruments, including the Short Portable Mental Status Questionnaire [12], the Mini-mental State Examination [13] and the Montreal Cognitive Assessment (MoCA) [14]. We chose the MoCA, a popular instrument for detecting mild cognitive impairment and dementia in clinical settings, because of its greater sensitivity to memory and its inclusion of items reflecting executive function $[15,16]$. We hypothesized that MoCA scores would be related inversely to Framingham Risk scores for coronary artery disease [17] and stroke [18], to older age and the presence of one or more apolipoprotein E4 (apoE4) alleles and would correlate directly with years of education.

We compared Framingham Coronary and Stroke risk scores obtained in 1999-2000 to MoCA scores obtained in 2008 and 2009.

\section{MATERIAL AND METHODS}

The Dallas Heart Study (DHS) is a population-based investigation designed to track the development of cardiovascular disease; $50 \%$ of the sample is African American [19]. The project, funded by the Donald W. Reynolds Foundation, was initiated in 1999. The first wave of examinees (DHS-1) who completed the entire 3-day study protocol (approximately 3000 subjects ranging in age from 30 to 65 years), was not adminis- 
tered a cognitive measure. DHS-2 examined 3,500 subjects, largely returnees from DHS-1. Cognitive screening was added to DHS-2 as part of a day-long visit that included extensive demographic and family history information, vital signs, EKG, measures of body fat, cardiac wall thickness, aortic plaque, coronary artery calcification, apoE genotype, and other measures. Additional DHS-2 measures included 3 Tesla MRI imaging of the brain, MRI determination of common carotid wall thickness, and a measure of depressive symptoms.

Framingham Coronary Risk and Stroke Risk scores were calculated from DHS-1 data. The Coronary Risk score is based on a formula including age, total cholesterol, cigarette smoking, high-density lipoprotein (HDL) concentration, and systolic blood pressure (see Table 1). Scores for men range from -10 to 37 ; for women, from -8 to 44 . When employed in an algorithm, these scores indicate the likelihood (as a percent risk) of a coronary event occurring within 10 years [20]. For this reason, we modified Coronary Risk scoring by converting negative scores to zero to create a continuous variable that could be compared with cognitive test scores.

Ten-year Framingham Stroke Risk scoring differs from Coronary Risk scoring in that it does not consider total or HDL cholesterol but does include diabetes, history of heart disease, atrial fibrillation, and left ventricular hypertrophy (LVH) (see Table 1). Scores range from zero to 48 points in men and 2 to 48 points in women. These scores indicate the likelihood (as a percent) of a cerebrovascular event occurring within 10 years [18]. For this study, we used the raw score for the Stroke Risk scale to create a continuous variable that could be compared with cognitive test scores.

The MoCA is a 30-point, 10 - 15 minute cognitive test that has been used primarily to detect mild cognitive impairment and dementia in clinical populations $[15,16]$. The MoCA samples a wide range of cognitive abilities, including orientation, attention, language, verbal memory, praxis, and mental flexibility. Existing norms are based on a Canadian sample $(\mathrm{N}=90)$ with a mean age in the mid 70s and mean education of approximately 12 years. Although less is known about its psychometric properties, the MoCA has a potential advantage over other brief cognitive screening tests such as the 30-item Mini-Mental State Examination (MMSE) [13] and the 10-item Short Portable Mental Status Questionnaire (SPMSQ) [12] because of its greater sensitivity to more subtle cognitive impairment [14,21].

Inclusion criteria: We included all subjects in the DHS-1 database fluent in English who had both Framingham Coronary and Stroke Risk scores and MoCA testing at the time of DHS-2.

Exclusion criteria: We excluded subjects with incom- plete Coronary and Stroke Risk scores or with a history of stroke or incomplete MoCA testing. All subjects in DHS-1 and DHS-2 studies signed informed consent documents approved by the UT Southwestern IRB.

Statistical methods:

For categorical variables, frequencies, percentages, and 95\% Confidence Intervals (95\%CI) were calculated. For continuous measures, means and standard deviations (mean \pm standard deviations) were calculated. The association between the continuous measures was examined using the Pearson Product Moment correlation and partial correlation coefficients. Two sets of multiple regression models predicting MoCA Total Scores from either Coronary Risk or Stroke Risk and included the covariates education and gender; gender was found to be non-significant in all models and was excluded from further modeling. Eight multiple regression models (four for each type of risk score) predicting MoCA Scores were fit to components of Coronary Risk and Stroke Risk scores individually. The unadjusted models included the components of each risk score and the adjusted models included education and gender in addition to the components of the risk score. For both types of models (adjusted and unadjusted), all components were included in a model (full model) followed by a stepwise procedure ( $p$ to enter and leave set at 0.05). Regression weights, 95\% confidence intervals (95\% CI), and R are reported for each model. No violations of the assumptions were found for any of the statistical tests performed. SPSS V18 was used in all analyses and the level of significance was set at $p<0.05$.

\section{RESULTS}

Educational level ranged from 0 (no formal schooling) to 20 years, with a mean of $12.3 \pm 2.3$ years. Age ranged from 18 to 65 years at the time of the first DHS-1 visit. MoCA scores ranged from 7 to 30 points and were available in 968 subjects; 952 of those also had Framingham Coronary Risk data available and 808 of these same subjects had Stroke Risk scores. The 23 subjects whose ethnicity was indicated as "Other" and the 20 subjects with a history of stroke were dropped from the analysis, leaving a total of 909 subjects with Coronary Risk scores and 765 of these same subjects with Stroke Risk scores. The ethnic composition of this sample was 49.9\% African American, 36.1\% Caucasian, and 14.0\% Hispanic; 42\% were men (Table 2). There were 16 subjects who completed the MoCA in Spanish, however, removing these 16 subjects from analyses did not affect results. The distribution of E4 alleles was $27.6 \%$ for one allele and $4.2 \%$ for two alleles, with an overall E4 allele frequency of $18.1 \%$.

For the group as a whole, there was a small but sig- 
Table 1. Framingham 10-year coronary and stroke risk variables.

\begin{tabular}{ccc}
\hline Variable & Coronary Risk & Stroke Risk \\
\hline Age & $\mathrm{X}$ & $\mathrm{X}$ \\
Systolic BP treated & $\mathrm{X}$ & $\mathrm{X}$ \\
Systolic BP untreated & $\mathrm{X}$ & $\mathrm{X}$ \\
Cigarettes & $\mathrm{X}$ & \\
Total cholesterol & $\mathrm{X}$ & $\mathrm{X}$ \\
HDL cholesterol & $\mathrm{X}$ & $\mathrm{X}$ \\
Diabetes & & $\mathrm{X}$ \\
Cardiovascular disease & & $\mathrm{X}$ \\
Atrial fibrillation & & \\
Left ventricular hypertrophy & & \\
\hline
\end{tabular}

Table 2. Categorical and continuous measures for coronary and stroke risk cohorts.

\begin{tabular}{|c|c|c|c|}
\hline \multirow[b]{2}{*}{ Measure } & \multirow[b]{2}{*}{ Value } & Coronary Risk $(\mathrm{N}=909)$ & \multirow[t]{2}{*}{ Stroke Risk $(\mathrm{N}=765)$} \\
\hline & & & \\
\hline \multirow[t]{3}{*}{ Ethnicity } & Black & $454(49.9 \%)$ & $366(47.8 \%)$ \\
\hline & White & $328(36.1 \%)$ & $294(38.4 \%)$ \\
\hline & Hispanic & $127(14.0 \%)$ & 105 (13.7\%) \\
\hline Gender & Male & $382(42.0 \%)$ & $324(42.4 \%)$ \\
\hline \multirow[t]{3}{*}{ ApoE4 Alleles } & 0 & $610(68.2 \%)$ & 515 (68.5\%) \\
\hline & 1 & 247 (27.6\%) & $210(27.9 \%)$ \\
\hline & 2 & $38(4.2 \%)$ & $27(3.6 \%)$ \\
\hline \multicolumn{2}{|c|}{ MoCA Score } & $23.42 \pm 3.95$ & $23.61 \pm 3.98$ \\
\hline \multicolumn{2}{|c|}{ Coronary Risk Score } & $9.84 \pm 5.29$ & $6.04 \pm 4.50$ \\
\hline \multicolumn{2}{|c|}{ Education (yrs) } & $13.45 \pm 2.95$ & $13.56 \pm 2.96$ \\
\hline \multicolumn{2}{|c|}{ Age (yrs) } & $51.43 \pm 9.58$ & $51.36 \pm 9.44$ \\
\hline \multicolumn{2}{|c|}{$\begin{array}{l}\text { Age (yrs) median, range } \\
\text { IRQ range }\end{array}$} & $\begin{array}{l}51.1,26.4-72.8 \\
\quad 44.3-58.8\end{array}$ & $\begin{array}{l}51,26.8-72.8 \\
\quad 4.4-58.6\end{array}$ \\
\hline
\end{tabular}

nificant inverse relationship between Coronary Risk scores in the DHS-1 cohort and MoCA scores obtained approximately 8 years later [ $(907)=-0.201, p<0.001$ ] Table 3 shows the multiple regression models used to predict MoCA scores from the components for Coronary and Stroke Risk Scores. When examining components of the Coronary Risk, score, age and systolic blood pressure were significant in the unadjusted full model while education and gender were significant in addition to age and systolic BP in the adjusted full model. The unadjusted stepwise model again resulted in both age and systolic BP found to be significant; however, only age and education were found significant in the adjusted stepwise model.

Of the 909 subjects with Coronary Risk scores, 765 also had Stroke Risk scores for comparison with MoCA scores. The ethnicity, gender, age and apoE4 allele distribution (allele frequency $=18.5 \%$ ) of this group was essentially the same as the Coronary Risk group (see
Table 2). For the group as a whole, there was again a significant correlation between MoCA and Stroke Risk scores $[r(763)=-0.215, p<0.001]$. On examining components of the Stroke Risk score, age, systolic blood pressure (BP), and left ventricular hypertrophy (LVH) were found to be significant in the unadjusted full model while systolic BP, LVH and education were found to be significant in the adjusted full model. The unadjusted stepwise model again resulted in age, systolic BP, and $\mathrm{LVH}$ found to be significant; however, only age, LVH and education were found significant in the adjusted stepwise model.

Controlling for the effect of education on MoCA scores, the partial correlation between MoCA scores and Framingham Coronary Risk and Stroke Risk scores was $r(905)=-0.205(p<0.001$ and $r(761)=-0.157(p<$ 0.001 ) respectively. Thus, after adjusting for education, a 1-point increase in Coronary Risk score was associated with a 0.138-point drop in MoCA score while a 1-point 
Table 3. Models predicting MoCA from components of coronary and stroke risk.

Coronary Risk

\begin{tabular}{|c|c|c|c|c|c|}
\hline \multirow[b]{2}{*}{ Model } & \multirow[b]{2}{*}{ Predictors } & \multicolumn{2}{|c|}{ Unadjusted Model Coefficients } & \multicolumn{2}{|c|}{ Adjusted Model Coefficients } \\
\hline & & B $(95 \% \mathrm{CI}) / \mathrm{R}$ & $p$-value & B $(95 \%$ CI $) / R$ & $p$-value \\
\hline 1 & Age & $-0.130(-0.187$ to -0.073$)$ & $<0.0001$ & $-0.148(-0.199$ to -0.096$)$ & $<0.0001$ \\
\hline \multirow[t]{7}{*}{ (Full) } & Cholesterol & $0.002(-0.126$ to 0.130$)$ & 0.9786 & $-0.008(-0.122$ to 0.106$)$ & 0.8944 \\
\hline & Smoker & -0.062 ( -0.171 to 0.047$)$ & 0.2639 & 0.035 (-0.063 to 0.133$)$ & 0.4845 \\
\hline & HDL & -0.008 ( -0.244 to 0.229$)$ & 0.9496 & 0.116 (-0.104 to 0.335$)$ & 0.3004 \\
\hline & Systolic BP & $-0.282(-0.460$ to -0.103$)$ & 0.0020 & $-0.222(-0.39$ to -0.054$)$ & 0.0095 \\
\hline & Education & -- & -- & 0.608 (0.531 to 0.686$)$ & $<0.0001$ \\
\hline & Gender (M) & -- & -- & $-0.683(-1.185$ to -0.18$)$ & 0.0078 \\
\hline & Model R & 0.225 & $<0.0001$ & 0.500 & $<0.0001$ \\
\hline 2 & Age & $-0.123(-0.177$ to -0.07$)$ & $<0.0001$ & $-0.176(-0.22$ to -0.131$)$ & $<0.0001$ \\
\hline \multirow[t]{3}{*}{ (Stepwise) } & Systolic BP & $-0.290(-0.466$ to -0.113$)$ & 0.0013 & -- & -- \\
\hline & Education & -- & -- & 0.603 (0.527 to 0.679 ) & $<0.0001$ \\
\hline & Model R & 0.222 & $<0.0001$ & 0.490 & $<0.0001$ \\
\hline
\end{tabular}

Stroke Risk

\begin{tabular}{|c|c|c|c|c|c|}
\hline \multirow[b]{2}{*}{ Model } & \multirow[b]{2}{*}{ Predictors } & \multicolumn{2}{|c|}{ Unadjusted Model Coefficients } & \multicolumn{2}{|c|}{ Adjusted Model Coefficients } \\
\hline & & B $(95 \% \mathrm{CI}) / \mathrm{R}$ & p-value & B (95\% CI)/R & p-value \\
\hline 1 & Age & $-0.236(-0.437$ to -0.036$)$ & 0.0211 & $-0.178(-0.361$ to 0.006$)$ & 0.0574 \\
\hline \multirow[t]{9}{*}{ (Full) } & Systolic BP & $-0.182(-0.33$ to -0.033$)$ & 0.0166 & $-0.137(-0.273$ to -0.001$)$ & 0.0490 \\
\hline & Diabetes & -0.256 ( -0.621 to 0.109$)$ & 0.1686 & $-0.138(-0.470$ to 0.195$)$ & 0.4171 \\
\hline & Smoker & -0.150 (-0.358 to 0.058$)$ & 0.1574 & $0.070(-0.122$ to 0.262$)$ & 0.4762 \\
\hline & CVD & 0.105 (-0.165 to 0.374$)$ & 0.4457 & 0.076 ( -0.170 to 0.321$)$ & 0.5459 \\
\hline & Afib & $0.095(-0.245$ to 0.435$)$ & 0.5835 & 0.055 ( -0.256 to 0.365$)$ & 0.7296 \\
\hline & LVH & -0.375 ( -0.591 to -0.158$)$ & 0.0007 & $-0.311(-0.509$ to -0.113$)$ & 0.0021 \\
\hline & Education & -- & -- & $0.556(0.470$ to 0.641$)$ & $<0.0001$ \\
\hline & Gender (M) & -- & -- & $-0.353(-0.862$ to 0.157$)$ & 0.1749 \\
\hline & Model R & 0.245 & $<0.0001$ & 0.477 & $<0.0001$ \\
\hline 2 & Systolic BP & $-0.204(-0.348$ to -0.059$)$ & 0.0057 & -- & -- \\
\hline \multirow[t]{4}{*}{ (Stepwise) } & LVH & $-0.379(-0.594$ to -0.163$)$ & 0.0006 & $-0.383(-0.567$ to -0.199$)$ & $<0.0001$ \\
\hline & Age & $-0.236(-0.433$ to -0.039$)$ & 0.0191 & $-0.240(-0.413$ to -0.067$)$ & 0.0067 \\
\hline & Education & -- & -- & 0.554 (0.471 to 0.638 ) & $<0.0001$ \\
\hline & Model R & 0.232 & $<0.0001$ & 0.469 & $<0.0001$ \\
\hline
\end{tabular}

increase in Stroke Risk score was associated with a 0.124-point decrease.

In order to further explore the potential role of age, the above analyses were repeated using data from the subset of 493 subjects aged 50 years or older in the Co- ronary Risk group and 413 subjects in the Stroke Risk group (mean age \pm standard deviation $=58.66 \pm 5.97$ years for the Coronary Risk group and $58.52 \pm 5.97$ years for the Stroke Risk group). There was a very small significant correlation between MoCA scores and Coro- 
nary Risk scores $[r(491)=-0.139, p=0.002]$ and Stroke Risk scores $[r(411)=-0.214, p<0.001]$ in these older subjects. Controlling for the effect of education on MoCA scores, the partial correlation between Framingham Coronary Risk and Stroke Risk scores in older subjects was $r(489)=-0.059(p=.191)$ and $r(409)=-0.110(p=$ 0.026 ) respectively. Thus, after adjusting for education in older subjects, a 1-point increase in Coronary Risk score was associated with a 0.067-point drop in MoCA score while a 1-point increase in Stroke Risk score was associated with a 0.082 -point decrease.

There were no significant differences in MoCA scores, Coronary Risk scores, or Stroke Risk scores for subjects with or without apoE4 alleles ( $p=0.110, p=0.312$, and $p=0.874$, respectively) in the entire cohort or among subjects age $50+(p=0.103, p=0.623$, and $p=0.526$, respectively). Also, there were no significant differences between men and women in the relationship between MoCA and Coronary or Stroke Risk scores when examined for the entire sample or the older group (data not shown).

\section{DISCUSSION}

The uniqueness of our study is that it examines vascular risk factors in a population-based sample that includes 50\% African Americans. It is possible that the risk factors we examined were not sufficiently sensitive to detect and quantify the effects of subclinical atherosclerosis. We have now begun exploring the relationship between more direct biological measures of atherosclerosis including the concentration of atherosclerosis-related inflammatory substances such as CRP and direct measures of atherosclerosis such as coronary artery calcium.

Our cognitive measure, the MoCA, was designed to be used in clinical settings in which there is great variation in cognitive function [22]. It has been suggested that, as a screening tool, it may have limited value in populations where prevalence of mild cognitive impairment is low [23]. However, the range of MoCA scores in this study was 7-30 (mean \pm standard deviation $=23.38 \pm 4$ ). Other investigators have found more robust relationships between cardiovascular disease and cognitive function using more detailed neurocognitive measures [24,25] and also with very crude measures. For example, one study found that SPMSQ scores were lower in the presence of apoE4 [26]. These subjects had lower initial SPMSQ scores, and there was increased disparity between E4 carriers and non-E4 carriers over a period of 4 years.

We found that the influence of Coronary or Stroke Risk scores on MoCA scores did not increase with age. Because the mean age of this study population was rela- tively young, it may be that the impact of coronary and stroke risk factors are limited at this age, indicating such patients either need continued follow up at a later time or more sensitive tests early on. We also did not find the negative effect of the apoE4 allele on cognition found in the another study [27] or in a meta-analysis of 77 studies in which apoE4 carriers performed more poorly on tests of global cognitive function, and the disparity between E4 and non-E4 carriers increased with age [28].

One study found significant interactions between the presence of E4 and verbal memory, verbal organization, nonverbal memory, set shifting and complex attention in a community-based group of subjects with an average age of 61 years, but systolic blood pressure was the only individual risk factor significantly related to these cognitive measures [25]. Because of the disparity of our findings from those of others in the literature, we reviewed data from non-demented older adults persons followed yearly at the UT Southwestern Alzheimer's Disease Center (ADC). We examined MMSE data from all 219 subjects who had both MMSE scores and apoE4 allele determination, of whom 81 (40\%) had one or more apoE4 alleles. We found no significant difference in MMSE scores in non-demented subjects with or without an apoE4 allele.

Our findings concerning the impact of coronary and stroke risk factors and E4 on cognition may be related to differences in the populations studied and in the psychological measure employed. Both our "young” (mean age $=51$ years) and our "old" (mean age $=58$ years) cohorts were relatively young in relation to the sample examined by Haan et al. [29] and the more recent meta-analytic study [28]. The relationship of apoE4 to cognition in other studies may be partially explainable by the possible inclusion in older populations of persons with incipient Alzheimer disease [30], which is less likely in our DHS sample. Another study, which examined the relationship of the apoE4 allele to MMSE scores in persons 659 persons followed over 22 years in a large community-based study, found no relationship between apoE4 status and MMSE scores, but there was a significant difference in delayed recall in persons $<65$ years of age [31]. They suggested survival bias as an explanation of the difference in apoE 4 influence on cognition.

Other studies have suggested that vascular disease influences performance on cognitive tasks associated with frontal lobe function more than those associated with other cortical areas [24]. The MoCA contains few items relating to this cognitive domain, and score ranges for those items are limited. Supporting this explanation is the finding that a delayed recall test was more sensitive than the MMSE in detecting cognitive decline in elders 
[31]. We plan to re-test a subgroup of DHS-2 subjects with additional brief measures that may increase sensitivity to executive and memory function and we will also follow them prospectively to examine the rate of cognitive change.

Although the MoCA samples many of the same cognitive areas than less sensitive instruments, several less sensitive instruments have shown stronger correlations of global cognitive function with cardiovascular risk factors, including age and apoE status. Our failure to find correlations of similar strength may be attributable to some limitation of the MoCA, the relative youth of the population we studied or differences between persons with presymptomatic and symptomatic cardiovascular disease.

\section{REFERENCES}

[1] Beeri, M.S., Ravona-Springer, R., Silverman, J.M. and Haroutunian, V. (2009) The effects of cardiovascular risk factors on cognitive compromise. Dialogues in Clinical Neuroscience, 11, 201-212.

[2] Middleton, L.E., Barnes, D.E., Lui, L.Y. and Yaffe, K. (2010) Physical activity over the life course and its association with cognitive performance and impairment in old age. Journal of the American Geriatrics Society, 58, 1322-1326. doi:10.1111/j.1532-5415.2010.02903.x

[3] Yaffe, K., Vittinghoff, E., Lindquist, K., et al. (2010) Posttraumatic stress disorder and risk of dementia among us veterans. Archives of General Psychiatry, 67, 608-613. doi:10.1001/archgenpsychiatry.2010.61

[4] Tzourio, C., Dufouil, C., Ducimetiere, P. and Alperovitch, A. (1999) Cognitive decline in individuals with high blood pressure: A longitudinal study in the elderly. Eva study group. Epidemiology of vascular aging. Neurology, 53, 1948-1952.

[5] Mielke, M. M., Rosenberg, P. B., Tschanz, J., et al. (2007) Vascular factors predict rate of progression in alzheimer disease. Neurology, 69, 1850-1858. doi:10.1212/01.wnl.0000279520.59792.fe

[6] Solomon, A., Kareholt, I., Ngandu, T., et al. (2007) Serum cholesterol changes after midlife and late-life cognition: Twenty-one-year follow-up study. Neurology, 68, 751-756. doi:10.1212/01.wnl.0000256368.57375.b7

[7] Yaffe, K., Barrett-Connor, E., Lin, F. and Grady, D. (2002) Serum lipoprotein levels, statin use, and cognitive function in older women. Archives of Neurology, 59, 378-384. doi:10.1001/archneur.59.3.378

[8] Reitz, C., Luchsinger, J., Tang, M.X., Manly, J. and Mayeux, R. (2005) Impact of plasma lipids and time on memory performance in healthy elderly without dementia. Neurology, 64, 1378-1383. doi:10.1212/01.WNL.0000158274.31318.3C

[9] Whitmer, R. A., Sidney, S., Selby, J., Johnston, S. C. and Yaffe, K. (2005) Midlife cardiovascular risk factors and risk of dementia in late life. Neurology, 64, 277-281. doi:10.1212/01.WNL.0000149519.47454.F2

[10] Schnaider Beeri, M., Goldbourt, U., Silverman, J.M., et al. (2004) Diabetes mellitus in midlife and the risk of de- mentia three decades later. Neurology, 63, 1902-1907.

[11] Yaffe, K., Kanaya, A., Lindquist, K., et al. (2004) The metabolic syndrome, inflammation, and risk of cognitive decline. Journal of the American Medical Association, 292, 2237-2242. doi:10.1001/jama.292.18.2237

[12] Pfeiffer, E. (1975) A short portable mental status questionnaire for the assessment of organic brain deficit in elderly patients. Journal of the American Geriatrics Society, 23, 433-441.

[13] Folstein, M.F., Folstein, S.E. and McHugh, P.R. (1975) "Mini-mental state". A practical method for grading the cognitive state of patients for the clinician. Journal of Psychiatric Research, 12, 189-198. doi:10.1016/0022-3956(75)90026-6

[14] Nasreddine, Z.S., Phillips, N. A., Bedirian, V., et al. (2005) The montreal cognitive assessment, moca: A brief screening tool for mild cognitive impairment. Journal of the American Geriatrics Society, 53, 695-699. doi:10.1111/j.1532-5415.2005.53221.x

[15] Smith, T., Gildeh, N. and Holmes, C. (2007) The montreal cognitive assessment: Validity and utility in a memory clinic setting. Canadian Journal of Psychiatry, 52, 329-332.

[16] Hoops, S., Nazem, S., Siderowf, A. D., et al. (2009) Validity of the MoCA and mmse in the detection of mci and dementia in parkinson disease. Neurology, 73, 1738-1745. doi:10.1212/WNL.0b013e3181c34b47

[17] D'Agostino, R. B., Sr., Grundy, S., Sullivan, L. M. and Wilson, P. (2001) Validation of the framingham coronary heart disease prediction scores: Results of a multiple ethnic groups investigation. Journal of the American Medical Association, 286, 180-187. doi:10.1001/jama.286.2.180

[18] D’Agostino, R.B., Wolf, P.A., Belanger, A.J. and Kannel, W.B. (1994) Stroke risk profile: Adjustment for antihypertensive medication. The framingham study. Stroke, 25, 40-43. doi:10.1161/01.STR.25.1.40

[19] Victor, R. G., Haley, R. W., Willett, D. L., et al. (2004) The dallas heart study: A population-based probability sample for the multidisciplinary study of ethnic differences in cardiovascular health. American Journal of Cardiology, 93, 1473-1480.

[20] Wilson, P.W., D’Agostino, R.B., Levy, D., et al. (1998) Prediction of coronary heart disease using risk factor categories. Circulation, 97, 1837-1847.

[21] Aggarwal, A. and Kean, E. (2010) Comparison of the folstein mini mental state examination (mmse) to the montreal cognitive assessment (MoCA) as a cognitive screening tool in an inpatient rehabilitation setting. Neuroscience and Medicine, 1, 39-42. doi:10.4236/nm.2010.12006

[22] Bernstein, I.H., Lacritz, L., Barlow, C.E., Weiner, M.F. and Defina, L. F. (2011) Psychometric evaluation of the montreal cognitive assessment (MoCA) in three diverse samples. Clinical Neuropsychology, 25, 119-126. doi:10.1080/13854046.2010.533196

[23] McLennan, S.N., Mathias, J.L., Brennan, L.C. and Stewart, S. (2010) Validity of the montreal cognitive assessment (MoCA) as a screening test for mild cognitive impairment (MCI) in a cardiovascular population. Journal of Geriatric Psychiatry and Neurology, 24, 33-38.

[24] Kuczynski, B., Jagust, W., Chui, H.C. and Reed, B. (2009) An inverse association of cardiovascular risk and frontal 
lobe glucose metabolism. Neurology, 72, 738-743. doi:10.1212/01.wnl.0000343005.35498.e5

[25] Zade, D., Beiser, A., McGlinchey, R., et al. (2010) Interactive effects of apolipoprotein $\mathrm{E}$ type 4 genotype and cerebrovascular risk on neuropsychological performance and structural brain changes. Journal of Stroke and Cerebrovascular Diseases, 19, 261-268.

doi:10.1016/j.jstrokecerebrovasdis.2009.05.001

[26] Fillenbaum, G.G., Landerman, L.R., Blazer, D.G., et al. (2001) The relationship of APOE genotype to cognitive functioning in older African-American and caucasian community residents. Journal of the American Geriatrics Society, 49, 1148-1155.

doi:10.1212/01.WNL.0000149643.91367.8A

[27] Blair, C.K., Folsom, A.R., Knopman, D.S., et al. (2005) APOE genotype and cognitive decline in a middle-aged cohort. Neurology, 64, 268-276. doi:10.1212/01.WNL.0000149643.91367.8A
[28] Wisdom, N.M., Callahan, J.L. and Hawkins, K.A. (2011) The effects of apolipoprotein e on non-impaired cognitive functioning: A meta-analysis. Neurobiology of Aging, 32, 63-74. doi:10.1001/jama.282.1.40

[29] Haan, M.N., Shemanski, L., Jagust, W.J., Manolio, T.A. and Kuller, L. (1999) The role of APOE epsilon4 in modulating effects of other risk factors for cognitive decline in elderly persons. Journal of the American Medical Association, 282, 40-46. doi:10.1001/jama.282.1.40

[30] Qiu, C. and Fratiglioni, L. (2010) Apolipoprotein E epsilon4 status and cognitive decline with and without dementia. Archives of Neurology, 67, 1036. doi:10.1001/archneurol.2010.163

[31] Kozauer, N.A., Mielke, M.M., Chan, G.K., Rebok, G.W. and Lyketsos, C.G. (2008) Apolipoprotein e genotype and lifetime cognitive decline. International Psychogeriatrics, 20, 109-123. doi:10.1017/S104161020700587X 